\title{
A scheme for direct detection of qubit-environment entanglement generated during qubit pure dephasing
}

\author{
Bartosz Rzepkowski ${ }^{1} \cdot K_{\text {Katarzyna Roszak }}{ }^{1}$ \\ Received: 30 July 2020 / Accepted: 9 November 2020 / Published online: 18 December 2020 \\ (c) The Author(s) 2020
}

\begin{abstract}
We propose a scheme for the detection of qubit-environment entanglement which requires only operations and measurements on the qubit, all within reach of current experimental state-of-the-art. The scheme works for any type of interaction which leads to pure dephasing of the qubit as long as the initial qubit state is pure. The scheme is direct in the sense that it allows the detection of entanglement present in the system at time $\tau$ after the initialization of the qubit in a superposition state. It requires a measurement on the qubit at time $\tau$ and a comparison of the post-measurement evolution to the evolution obtained by a modified scheme. It becomes particularly simple when one of the qubit states is neutral with respect to the environment, such as in case of the most common choice of the NV center spin qubit or for excitonic charge qubits, when the environment is initially at thermal equilibrium. In this case, the postmeasurement evolution needs to be compared only to the standard decoherence which is obtained without any qubit manipulation after the preparation of the initial state.
\end{abstract}

Keywords Entanglement · Open quantum systems · Decoherence

\section{Introduction}

The accessibility of entanglement in larger bipartite systems is very limited up to date, because contrarily to entanglement between two qubits [1-3], the theoretical means for the study of such entanglement are very limited unless the joint system state is pure. The only available measure which can be calculated from the density matrix is negativity [4,5] or closely related logarithmic negativity [6], the calculation of which requires diagonalization of a matrix of the same dimension as the joint Hilbert space of the two parties, which must be and has been done numerically [7-10]. This

Katarzyna Roszak

katarzyna.roszak@pwr.edu.pl

1 Department of Theoretical Physics, Wrocław University of Science and Technology, 50-370

Wrocław, Poland 
limits the range of general conclusions which can be reached about the creation and behavior of entanglement. Experimentally, such entanglement is hardly accessible at all, since measuring negativity would require full quantum-state tomography, similarly as quantification of two-qubit entanglement, but as the technique can be done for two small systems [11-13], it exceeds the current experimental state-of-the-art once either of the potentially entangled systems becomes large.

The problem is that the question of entanglement becomes important when dealing with decoherence between a quantum system of interest, such as a qubit, and its environment. This is because the presence of entanglement, although its manifestation is limited when straightforward qubit decoherence is of interest $[14,15]$, can significantly change the effect that the environment has on the system in more involved procedures and algorithms, especially ones that involve qubit evolution post-measurement [1623].

The question of entanglement generation becomes more solvable once limitations on the generality of the problem are imposed. It has recently been shown that for evolutions which lead to pure dephasing of a qubit or even a larger system [24-28], there exists a straightforward signature, which entanglement leaves on the state of the environment [24,25] (after the qubit/system state is traced out). This is, on the one hand, the reason why it is important to know if qubit-environment entanglement (QEE) is generated, since only decoherence with generation of QEE is accompanied by the information about the qubit state leaking out into the environment [29], similarly as in the case of a pure environment [30,31]. On the other hand, it also serves as the basis for the possibility of direct measurement of QEE.

In the following, we describe a scheme for the direct experimental detection of QEE which occurs at time $\tau$ after the creation of a qubit superposition state. The scheme works only within the class of Hamiltonians that leads to qubit pure dephasing, but it involves operations and measurements performed only on the qubit. The required operations are well within reach of all systems that have been proposed as qubits, especially in the solid state, where pure dephasing is commonly the dominant source of decoherence [32-40]. The scheme relies on the fact that although it is the state of the environment which is distinctly affected by the presence of QEE, it can in turn influence the evolution of the qubit.

The scheme differs significantly from the one presented in Ref. [41], as the operations and measurements on the qubit required are different. Hence, the scheme will be more appropriate for different qubit realizations depending on the experimental stateof-the-art. Furthermore, the scheme detects qubit-environment entanglement present in the state contrarily to the scheme of Ref. [41], which serves to test if entanglement would be present at a given time, for the same initial state of the environment if the initial state of the qubit were a superposition and not one of the pointer states which are used in the scheme.

The scheme presented here is especially well-suited for interactions which are asymmetric in the sense that only one of the qubit states couples to the environment, while the other remains unaffected by it. In this case, when the environment is initially at thermal equilibrium, the procedure becomes particularly simple, because detecting entanglement requires only the comparison of one part of the general procedure with regular decoherence curves (when no additional preparation is performed on the qubit 
prior to exciting a superposition state). Such qubit-environment systems are common; it is the case for the most common choice of NV center spin qubit interacting with a spin environment [42-44], as well as for excitonic charge qubits interacting with phonons [45-48]. We exemplify the working of the scheme on the latter qubit system.

The paper is organized as follows. In Sect. 2, we introduce the class of systems studied and the entanglement witness which is applicable for such systems. The proposed experimental scheme is described in Sect. 3. The simplified measurement procedure which is applicable if the class of interactions is further limited is introduced in Sect. 3.4. In Sect. 4, we provide exemplary results for the workings of the scheme, and Sect. 5 concludes the paper.

\section{The interaction}

We will consider a system consisting of a single qubit (Q) in the presence of an arbitrary environment (E). The most general form of a QE Hamiltonian that leads to qubit pure dephasing is given by

$$
\hat{H}=\sum_{i=0,1} \epsilon_{i}|i\rangle\left\langle i\left|+\hat{H}_{E}+\sum_{i=0,1}\right| i\right\rangle\langle i| \otimes \hat{V}_{i}
$$

The first part of the Hamiltonian characterizes the qubit. For no processes involving energy exchange between $\mathrm{Q}$ and $\mathrm{E}$ to take place and therefore for the interaction to lead to pure dephasing, it must commute with the last, interaction, term of the Hamiltonian. $\hat{H}_{E}$ represents the free Hamiltonian of the environment and is arbitrary. The last term specifies the qubit-environment interaction with the qubit states written on the left side of the tensor product. For now, we do not impose any restrictions on the components occurring in Eq. (1), so the interaction it describes is of most general form.

The evolution operator of the QE system resulting from the Hamiltonian (1) can be formally written as

$$
\hat{U}(t)=\exp \left(-\frac{i}{\hbar} \hat{H} t\right)=\left[\begin{array}{cc}
\hat{w}_{0}(t) & 0 \\
0 & \hat{w}_{1}(t)
\end{array}\right]
$$

where the matrix form is kept in terms of qubit pointer states $|0\rangle$ and $|1\rangle$, while the evolution of the environment is described by the operators $\hat{w}_{i}(t), i=0,1$. These operators are given by

$$
\hat{w}_{i}(t)=\exp \left(-\frac{i \epsilon_{i} t}{\hbar}\right) \exp \left(-\frac{i}{\hbar}\left(\hat{H}_{E}+\hat{V}_{i}\right) t\right)
$$

Note, that we could achieve this concise form only because the free qubit Hamiltonian commutes with all other Hamiltonian terms. 


\subsection{Criterion of qubit-environment entanglement}

It is shown in Ref. [24] that the QE system initially in a product state and undergoing evolution for time $\tau$ governed by (2) is separable, iff

$$
\left[\hat{w}_{0}^{\dagger}(\tau) \hat{w}_{1}(\tau), \hat{R}(0)\right]=0
$$

Here, $\hat{R}(0)$ denotes an arbitrary initial state of the environment. The qubit initial state has to be pure and a superposition of both pointer states. If we introduce the following notation

$$
\hat{R}_{i j}(\tau)=\hat{w}_{i}(\tau) \hat{R}(0) \hat{w}_{j}^{\dagger}(\tau),
$$

with $i, j=0,1$, we can reformulate the QEE criterion of Eq. (4) to say that the QE state is entangled at time $\tau$ iff

$$
\hat{R}_{00}(\tau)=\hat{R}_{11}(\tau)
$$

\section{The scheme}

The proposed scheme for detection of QEE relies on the fact that the state of the environment influences the state of the qubit and similarly the state of the qubit influences the state of the environment throughout their joint evolution. Hence, even though the presence of QEE leaves a detectable mark only on the state of the environment during a simple joint evolution, it is possible to measure this effect when only the qubit is accessible. A fully indirect scheme for the detection of QEE has been recently proposed in Ref. [41], where in fact the possibility of a given QE system to become entangled was tested, rather than the entanglement present in the system during the potential experiment.

Here, we show a method which allows to test the presence of entanglement at a given time $\tau$ by further processing and measuring the state of the qubit and later comparing the post- $\tau$ evolution of the qubit with results obtained in a test run of the same initial qubit and environment state.

\subsection{Evolution of the system with intermediate measurement at time $\tau$}

Let us assume that the initial state of the qubit is an equal superposition state, $|+\rangle=$ $(|0\rangle+|1\rangle) / \sqrt{2}$, so that the QE initial state is given by $\hat{\sigma}(0)=|+\rangle\langle+| \otimes \hat{R}(0)$. In the first part of the scheme, we allow the two subsystems to undergo simple joint evolution as governed by the Hamiltonian (1) until time $\tau$,

$$
\hat{\sigma}(\tau)=\hat{U}(\tau) \hat{\sigma}(0) \hat{U}^{\dagger}(\tau)=\frac{1}{2}\left[\begin{array}{ll}
\hat{R}_{00}(\tau) & \hat{R}_{01}(\tau) \\
\hat{R}_{10}(\tau) & \hat{R}_{11}(\tau)
\end{array}\right] .
$$

Time $\tau$ is singled out as the time at which we are testing QEE. In other words, a positive result of the proposed scheme would certify that there is entanglement in state (7). 
Incidentally, it is rather straightforward to generalize the proposed scheme to any initial qubit state, but if QEE is generated for state $|+\rangle$ then it would also be generated for any superposition of pointer states [24], $|\psi\rangle=a|0\rangle+b|1\rangle$, with $a, b \neq 0$, so there is hardly any point.

The first step toward determining QEE in state (7) is to measure the qubit in the $\{|+\rangle,|-\rangle\}$ basis, where the $|+\rangle$ state is the initial state of the qubit and the $|-\rangle$ state is orthogonal to it. A projective measurement in the qubit subspace yields the states $| \pm\rangle$ with probabilities

$$
p_{ \pm}(\tau)=\frac{1}{4} \operatorname{Tr}\left(\hat{R}_{00}(\tau) \pm \hat{R}_{01}(\tau) \pm \hat{R}_{10}(\tau)+\hat{R}_{11}(\tau)\right)
$$

Although indirectly, the measurement also influences the environment while it leads to the recurrence of a product QE state, with new environmental states depending on the outcome of the measurement,

$$
\hat{R}_{ \pm}(\tau)=\frac{\hat{R}_{00}(\tau) \pm \hat{R}_{01}(\tau) \pm \hat{R}_{10}(\tau)+\hat{R}_{11}(\tau)}{4 p_{ \pm}(\tau)}
$$

Using $\hat{R}_{ \pm}(\tau)$ we can write the post-measurement QE states at time $\tau$ as $\hat{\sigma}_{ \pm}(\tau)=$ $| \pm\rangle\langle \pm| \otimes \hat{R}_{ \pm}(\tau)$. Since the Hamiltonian remains unchanged, it is straightforward to find the evolution which occurs after additional time $t$ post-measurement has passed,

$$
\hat{\sigma}_{ \pm}(\tau+t)=\frac{1}{2}\left[\begin{array}{cc}
\hat{w}_{0}(t) \hat{R}_{ \pm}(\tau) \hat{w}_{0}^{\dagger}(t) & \pm \hat{w}_{0}(t) \hat{R}_{ \pm}(\tau) \hat{w}_{1}^{\dagger}(t) \\
\pm \hat{w}_{1}(t) \hat{R}_{ \pm}(\tau) \hat{w}_{0}^{\dagger}(t) & \hat{w}_{1}(t) \hat{R}_{ \pm}(\tau) \hat{w}_{1}^{\dagger}(t)
\end{array}\right]
$$

The quantity, which will later allow us to distinguish whether the pre-measurement state at time $\tau$ (7) is entangled or separable is the evolution of the qubit coherence. To this end, we find the post-measurement evolution of the qubit by tracing out environmental degrees of freedom (the evolution of the qubit is of pure-dephasing type, so only off-diagonal elements of the qubit density matrix evolve) and the coherence is given by

$$
\rho_{ \pm}^{01}(\tau+t)= \pm \frac{1}{2} \operatorname{Tr}\left(\hat{w}_{0}(t) \hat{R}_{ \pm}(\tau) \hat{w}_{1}^{\dagger}(t)\right)
$$

The last step involves averaging the qubit coherence over the measurement outcomes, so the quantity of interest which, as it will turn out, contains information allowing to determine entanglement, is given by

$$
\rho_{a v}^{01}(\tau+t)=p_{+}(\tau) \rho_{+}^{01}(\tau+t)-p_{-}(\tau) \rho_{-}^{01}(\tau+t) .
$$

During the averaging, the minus sign stemming from the coherence of the initial qubit state $|-\rangle$ is compensated for [hence the difference and not the sum in the second term of Eq. (12)]. Experimentally, this means that the same procedure, involving preparation of the initial qubit equal superposition state, allowing it to evolve for time $\tau$, after which a measurement of the qubit is performed in the $\{| \pm\rangle\}$ basis, and the following 
measurement of the evolution of qubit coherence, needs to be repeated a sufficient number of times, and the results have to be averaged regardless of the measurement outcome. Inserting explicit formulas for the probabilities of each measurement outcome (8) and the corresponding coherences (11) into Eq. (12), we get a much simpler formula than the one for the coherences alone,

$$
\rho_{a v}^{01}(\tau+t)=\frac{1}{4} \operatorname{Tr}\left[\hat{w}_{0}(t)\left(\hat{R}_{00}(\tau)+\hat{R}_{11}(\tau)\right) \hat{w}_{1}^{\dagger}(t)\right] .
$$

\subsection{Evolution of the comparative system}

To determine if there is entanglement in state (7), the quantity (13) needs to be compared to the outcome of a second procedure. We will later show that in the most common scenario, this second procedure reduces to a straightforward measurement of coherence with no additional preparation.

Contrary to the first part, the qubit is initialized in state $|0\rangle$ with the environment in the same state as before, $\hat{\sigma}_{0}(0)=|0\rangle\langle 0| \otimes \hat{R}(0)$. As previously, we allow the QE system to evolve jointly for time $\tau$, which leads to no change in the qubit state, but does lead to an evolution in the subspace of the environment, $\hat{\sigma}_{0}(\tau)=|0\rangle\langle 0| \otimes \hat{R}_{00}(\tau)$, where $\hat{R}_{00}(\tau)$ is given by Eq. (5). At time $\tau$ instead of conducting a measurement, the superposition $|+\rangle$ is excited in the qubit subspace. The later QE evolution as a function of time $t$ (time elapsed after the excitation) differs from the undisturbed QE evolution only by the initial state of the environment. Here, the quantity of interest is again the coherence of the qubit as a function of time $t$ which is obtained after tracing out the degrees of freedom of the environment and is given by

$$
\rho_{0}^{01}(\tau+t)=\frac{1}{2} \operatorname{Tr}\left(\hat{w}_{0}(t) \hat{R}_{00} \hat{w}_{1}^{\dagger}(t)\right)
$$

\subsection{The quantity of interest}

To detect QEE, we need to study the difference between the average qubit coherence obtained from the procedure involving an intermediate measurement (13) and the coherence of the comparative system (14),

$$
\Delta \rho^{01}(\tau+t)=\rho_{a v}^{01}(\tau+t)-\rho_{0}^{01}(\tau+t)=\frac{1}{4} \operatorname{Tr}\left[\hat{w}_{0}(t)\left(\hat{R}_{11}(\tau)-\hat{R}_{00}(\tau)\right) \hat{w}_{1}^{\dagger}(t)\right] .
$$

Obviously it can be nonzero only if $\hat{R}_{11}(\tau) \neq \hat{R}_{00}(\tau)$, hence the quantity can be nonzero only if there is entanglement in state (7), and it is therefore an entanglement witness. In fact, if the difference of coherences (15) is nonzero at any time $t$ then there must have been QEE in the pre-measurement state at time $\tau$.

Otherwise, either the QE state (7) was separable and $\hat{R}_{11}(\tau)=\hat{R}_{00}(\tau)$ or the conditional environmental evolution operators (3) commute with one another. In case of commutation (the condition of commutation is $\left[\hat{H}_{E}+\hat{V}_{0}, \hat{H}_{E}+\hat{V}_{1}\right]=0$ ) we have 


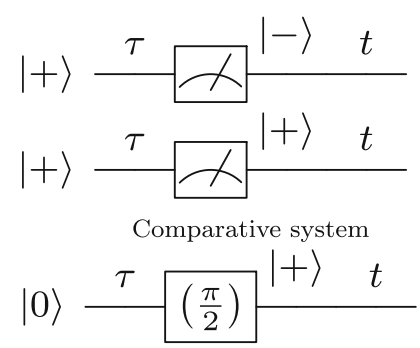

Fig. 1 Graphical representation of the general protocol for QEE detection with intermediate measurement at time $\tau$

$$
\operatorname{Tr}\left[\hat{w}_{0}(t) \hat{R}_{i i}(\tau) \hat{w}_{1}^{\dagger}(t)\right]=\operatorname{Tr}\left[\hat{w}_{0}(t) \hat{R}(0) \hat{w}_{1}^{\dagger}(t)\right]
$$

for $i=0,1$ and this type of entanglement cannot be detected using the scheme under study. In fact, the class of entangled states which will not be detected by this scheme are exactly the same as the class not detected by the scheme described in Ref. [41]. For details on why schemes for detecting QEE where operations and measurements are restricted to the qubit subspace will not be able to detect entanglement generated by conditional evolution operators which commute see Appendix. The protocol is shown in Fig. 1.

\subsection{Asymmetric interaction}

The true advantage of the scheme described here lies in the situation when the interaction between the qubit and its environment is asymmetric, $\hat{V}_{0}=\mathbb{I}$, and the single nontrivial interaction term does not commute with the free Hamiltonian of the environment, $\left[\hat{H}_{E}, \hat{V}_{1}\right] \neq 0$ (the latter condition is necessary so that the conditional evolution operators acting on the environment do not commute). This situation is reasonably common for solid-state qubits, for which pure-dephasing evolutions are the most common source of decoherence, since it means that one of the qubit states does not interact with the environment. This is the case for e. g. excitonic charge qubits interacting with phonons, in which case the $|0\rangle$ state consists of no exciton [45-48].

If additionally the initial state of the environment is some function of the free Hamiltonian $\hat{H}_{E}$, then the whole second part of the protocol is superfluous, and a comparison of the results of the first part of the procedure with a straightforward measurement of the evolution of coherence of an initial $|+\rangle$ qubit state as a function of time $t$ is enough to determine if the state (7) is entangled. This is because within the specified constraints $\left[\hat{w}_{0}(\tau), \hat{R}(0)\right]=0$, since $\hat{w}_{0}(\tau)$ and $\hat{R}(0)$ are functions of the same part of the Hamiltonian, namely $\hat{H}_{E}$. This means that $\hat{R}_{00}(\tau)=\hat{R}(0)$ and no extra preparation time in the comparative evolution is necessary.

The situation when the initial state of the environment is a function of the free Hamiltonian of the environment is the most common of all experimentally encountered situations, since any environment at thermal equilibrium falls into this category. The only situations when this does not apply, are when the environment has been spe- 


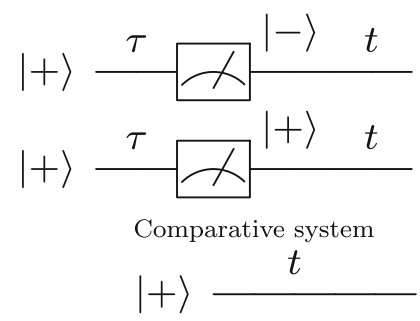

Fig. 2 Graphical representation of the simplified protocol for the system with asymmetric interaction

cially prepared by prior schemes, such as dynamical polarization [49-55]. Hence, for a qubit for which one of the pointer states is neutral with respect to the environment, and an environment initially at thermal equilibrium, the scheme for entanglement detection significantly simplifies. In fact, it is enough to compare results of the evolution of decoherence post an intermediate measurement in the equal superposition basis averaged over the possible measurement outcomes (12) with the plain evolution of decoherence, and the quantity of interest (15) simplifies to

$$
\Delta \rho^{01}(\tau+t)=\rho_{a v}^{01}(\tau+t)-\rho^{01}(t),
$$

where $\rho^{01}(t)=\left\langle 0\left|\operatorname{Tr}_{E} \hat{\sigma}(t)\right| 1\right\rangle$, and $\hat{\sigma}(t)$ is given by Eq. (7) with argument $t$ instead of $\tau$. The simplified protocol is illustrated in Fig. 2 .

\section{Example: charge qubit and phonons}

In the following, we will exemplify the validity and huge sensitivity of the procedure on an excitonic quantum dot qubit interacting with a bath of bulk phonons, the evolution of which is known to be entangling [10]. The setup and procedure is exactly as in Ref. [21]. The qubit state $|0\rangle$ corresponds to an empty quantum dot, while qubit state $|1\rangle$ is a ground state exciton confined in the dot, so the interaction is naturally asymmetric and the simplified procedure applies. The Hamiltonian of the system is given by

$$
\hat{H}=\varepsilon|1\rangle\left\langle 1\left|+\sum_{k} \hbar \omega_{k} \hat{b}_{k}^{\dagger} \hat{b}_{k}+\right| 1\right\rangle\langle 1| \otimes \sum_{k}\left(f_{k}^{*} \hat{b}_{k}+f_{k} \hat{b}_{k}^{\dagger}\right)
$$

where $\varepsilon$ is the energy of the exciton, $\hbar \omega_{k}$ are phonon energies corresponding to phonon creation and annihilation operators for wavevector $\boldsymbol{k}, \hat{b}_{\boldsymbol{k}}^{\dagger}$ and $\hat{b}_{\boldsymbol{k}} \cdot f_{\boldsymbol{k}}$ in the interaction term denote the deformation potential coupling constants [56,57], which is the dominating interaction leading to exciton decoherence. They are given by

$$
f_{\boldsymbol{k}}=\left(\sigma_{\mathrm{e}}-\sigma_{\mathrm{h}}\right) \sqrt{\frac{\hbar k}{2 \varrho V_{\mathrm{N}} c}} \int_{-\infty}^{\infty} d^{3} \boldsymbol{r} \psi^{*}(\boldsymbol{r}) e^{-i \boldsymbol{k} \cdot \mathrm{r}} \psi(\boldsymbol{r}) .
$$


Here, $\varrho$ is the crystal density, $V_{\mathrm{N}}$ is the normalization volume of the phonon system, $\sigma_{e}$ and $\sigma_{h}$ are deformation potential constants for electrons and holes, and $\psi(\boldsymbol{r})$ are excitonic wave functions.

The Hamiltonian can be diagonalized exactly by means of the Weyl operator method $[56,58]$. The exciton-phonon interaction term in the Hamiltonian is linear in phonon operators and describes a shift of the lattice equilibrium induced by the presence of a charge distribution in the dot. The stationary state of the system corresponds to the exciton being surrounded by a coherent cloud of phonons (representing the lattice distortion to the new equilibrium). The transformation that creates the coherent cloud is the shift

$$
\hat{w} \hat{b}_{k} \hat{w}^{\dagger}=\hat{b}_{k}-\frac{f_{k}}{\hbar \omega_{k}},
$$

generated by the Weyl operator [48] given by

$$
\hat{w}=\exp \left[\sum_{\boldsymbol{k}}\left(\frac{f_{\boldsymbol{k}}}{\hbar \omega_{k}} \hat{b}_{\boldsymbol{k}}^{\dagger}-\frac{f_{\boldsymbol{k}}^{*}}{\hbar \omega_{\boldsymbol{k}}} \hat{b}_{\boldsymbol{k}}\right)\right] .
$$

A straightforward calculation shows that the Hamiltonian is diagonalized by the unitary transformation

$$
\mathbb{W}=|0\rangle\langle 0|\otimes \mathbb{I}+| 1\rangle\langle 1| \otimes \hat{w},
$$

where $\mathbb{I}$ is the identity operator and the tensor product refers to the carrier subsystem (first component) and its phonon environment (second component).

This allows us to find the explicit form of conditional evolution operators acting on the environment, Eq. (3),

$$
\begin{aligned}
& \hat{w}_{0}(t)=e^{-\frac{i}{\hbar} \sum_{k} \hbar \omega_{k} \hat{b}_{k}^{\dagger} \hat{b}_{k} t}, \\
& \hat{w}_{1}(t)=e^{-\frac{i}{\hbar} E t} \hat{w}^{\dagger} \hat{w}_{0}(t) \hat{w} .
\end{aligned}
$$

The diagonalization procedure induces a shift in the energy of the exciton, which is now given by

$$
E=\varepsilon-\sum_{k} \frac{\left|f_{k}\right|^{2}}{\hbar \omega_{k}} .
$$

The explicit forms of the $\hat{w}_{i}(t)$ operators and the rules for multiplying Weyl operators [48] allow us to first find the conditional density matrices of the environment $R_{i i}(\tau)$ using Eq. (5) and then the quantity of interest (17), which is given by

$$
\begin{aligned}
\Delta \rho^{01}(t)= & \frac{1}{4} e^{-\frac{i}{\hbar} E t} e^{i \sum_{k}\left|\frac{f_{k}}{\hbar \omega_{k}}\right|^{2} \sin \omega_{k} t} e^{-\sum_{k}\left|\frac{f_{k}}{\hbar \omega_{k}}\right|^{2}\left(1-\cos \omega_{k} t\right)\left(2 n_{k}+1\right)} \\
& \times\left[e^{2 i \sum_{k}\left|\frac{f_{k}}{\hbar \omega_{k}}\right|^{2} \sin \omega_{k} \tau}-1\right] .
\end{aligned}
$$

The two phase terms dependent on time $t$ in the first line of Eq. (26) are irrelevant and can be easily eliminated by taking the absolute value. The real term in this line governs 


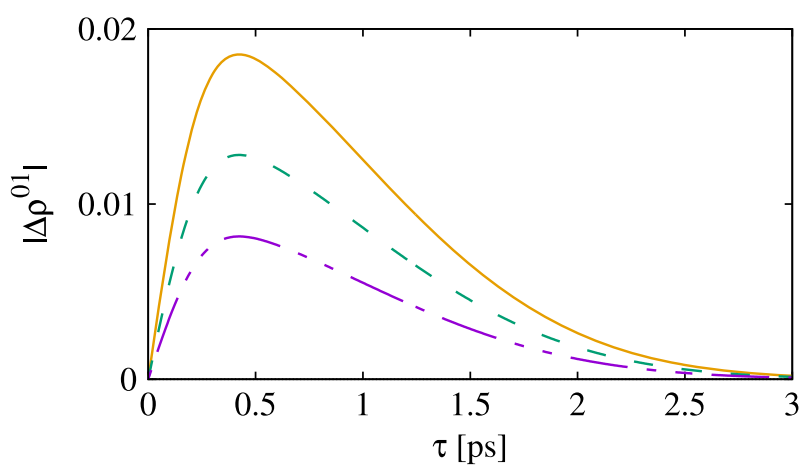

Fig. $3 \tau$ dependence of the measurable QEE witness (17) for a quantum dot excitonic qubit interacting with a phonon bath at $t \rightarrow \infty$ for different temperatures: $T=0 \mathrm{~K}$-yellow solid line; $T=34 \mathrm{~K}$-green dashed line; $T=70 \mathrm{~K}$-purple dashed-dotted line

the degree of decoherence and it guarantees that no entanglement will be signified for infinite temperature, when the initial density matrix of the environment is proportional to unity and pure dephasing evolutions cannot lead to entanglement [24]. Note that since the bath is super-Ohmic, we are dealing with partial pure dephasing [45-48] and for long times, the degree of coherence stabilizes at a certain, nonzero value instead of tending to zero. The most important term for the detection of entanglement is given by the second line of Eq. (26). The $\tau$ dependent phase is the signature of entanglement and it is similar in nature to the phase reported in Ref. [59], which signified the quantumness of the environment.

Figure 3 shows the $\tau$-dependence of the QEE witness given by the expression (17) in the limit $t \rightarrow \infty$ for material parameters characteristic for small GaAs quantum dots and bulk phonon modes [21,32] for different temperatures. The presented results were obtained by modeling single particle (electron and hole, which form the exciton) wave functions $\psi(\boldsymbol{r})$ as Gaussians with $5 \mathrm{~nm}$ width in the $x y$ plane and $1 \mathrm{~nm}$ along $z$. The material parameters used are $\sigma_{\mathrm{e}}-\sigma_{\mathrm{h}}=9 \mathrm{eV}, \varrho=5360 \mathrm{~kg} / \mathrm{m}^{3}$, and $c=5100$ $\mathrm{m} / \mathrm{s}$. The shifted exciton energy is not needed (but would have been of the order of 1 $\mathrm{eV})$.

The effect is small as to be expected, since the environment taken into account is very large, which yields very small amounts of entanglement [10]. Note that contrary to the results in [21], the effect is most pronounced when the intermediate measurement at time $\tau$ occurs before equilibration (which for the studied system is after around 3 ps). The quantity (17) is proportional to how different the conditional states of the environment are from each other, so it is proportional to the amount of QEE [29]. In fact, it is testimony to the extreme sensitivity of the method that it is visible at all.

\section{Conclusion}

We have proposed a scheme for the detection of QEE at a certain time $\tau$ which requires the comparison of qubit evolution after a measurement performed at said time $\tau$ with 
a second, similarly simple procedure performed also only on the qubit. The procedure is qualitatively different from the one proposed before [41] in the fact that it detects entanglement actually present in the system during its operation, when the results are compared to the ones obtained during another measurement. The characteristic feature of the procedure is that it requires an intermediate projective measurement so that information about the qubit state is transferred into the environment. This process is sensitive to qubit-environment entanglement, and the post-measurement evolution is different when the pre-measurement evolution was entangling.

The method is applicable to any qubit-environment interaction which leads to qubit pure dephasing, but is particularly useful, if the qubit-environment interaction is asymmetric (so that one of the qubit pointer states does not interact with the environment) and the initial state of the environment is at thermal equilibrium. Then, the procedure becomes particularly simple, and the post-measurement evolution needs only to be compared to the plain decoherence of the qubit. This means that if the evolution of qubit coherence post-measurement differs in any way from the evolution which would be observed if a superposition state was simply excited at the measurement time, then there must have been entanglement between the qubit and the environment pre-measurement at time $\tau$.

The method is of both experimental and theoretical interest, as it requires the description only of qubit evolution and does not require the knowledge of the explicit state of the environment apart from the initial state. As an example we have studied the presence of entanglement between a charge qubit confined in a quantum dot with an environment of bulk phonons at finite temperature. The evolution is known to be entangling, but very weakly so; nevertheless, we have observed a distinct signature of qubit-environment entanglement.

Acknowledgements K. R. acknowledges support from funds of the Polish National Science Center (NCN), Grant No. 2015/19/B/ST3/03152.

\section{Compliance of ethical standards}

Conflict of interest The authors declare that they have no conflict of interest.

Open Access This article is licensed under a Creative Commons Attribution 4.0 International License, which permits use, sharing, adaptation, distribution and reproduction in any medium or format, as long as you give appropriate credit to the original author(s) and the source, provide a link to the Creative Commons licence, and indicate if changes were made. The images or other third party material in this article are included in the article's Creative Commons licence, unless indicated otherwise in a credit line to the material. If material is not included in the article's Creative Commons licence and your intended use is not permitted by statutory regulation or exceeds the permitted use, you will need to obtain permission directly from the copyright holder. To view a copy of this licence, visit http://creativecommons.org/licenses/by/4.0/.

\section{A Commuting conditional evolution operators of the environment}

In this appendix we consider, why QEE with commuting conditional evolution operators of the environment is not likely to be distinguishable by operations and measurements on the qubit alone. The conditional operators are given in the Interac- 
tion section by Eq. (3). The evolution is separable at time $\tau$ iff the separability criterion (6) is fulfilled, which written explicitly with the help of the $\hat{w}_{0}(\tau)$ operators and the initial state of the environment, $\hat{R}(0)$, takes the form

$$
\hat{w}_{0}(\tau) \hat{R}(0) \hat{w}_{0}^{\dagger}(\tau)=\hat{w}_{1}(\tau) \hat{R}(0) \hat{w}_{1}^{\dagger}(\tau)
$$

otherwise, it is entangled [24]. We distinguish and study here a type of entangled evolution for which the conditional evolution operators of the environment commute at any two times,

$$
\left[\hat{w}_{0}\left(t_{0}\right), \hat{w}_{1}^{\dagger}\left(t_{1}\right)\right]=0 .
$$

Such commutation is a direct consequence of the commutation of the relevant parts of the Hamiltonian which drive the evolution of the environment, if the qubit is respectively in state $|0\rangle$ or $|1\rangle$,

$$
\left[\hat{H}_{E}+\hat{V}_{0}, \hat{H}_{E}+\hat{V}_{1}\right]=0 \text {. }
$$

In the following, we will call this type of entangling evolution "weak."

All methods that we can envision which serve to distinguish entangling from nonentangling evolutions are based (more or less directly) on the comparison of terms which are at most combinations (probably linear superpositions) of terms of type

$$
r(t)=\operatorname{Tr}\left[\hat{w}_{i_{1}}\left(t_{1}\right) \ldots \hat{w}_{i_{N}}\left(t_{N}\right) \hat{R}(0) \hat{w}_{j_{N}}^{\dagger}\left(t_{N}\right) \ldots \hat{w}_{j_{1}}^{\dagger}\left(t_{1}\right)\right] .
$$

This is the case in the scheme proposed here as well as in the scheme of ref. [41].

If we choose two functions of the type given by Eq. (30) which differ only by $n$ operators $\hat{w}_{i}(t)$ symmetrically on the left and right of $\hat{R}(0)$ which are closest to $\hat{R}(0)$, these two functions must yield the same results, if the separability criterion (27) is fulfilled for all relevant times. But operators under the trace can be cyclically permuted, so

$$
r(t)=\operatorname{Tr}\left[\hat{w}_{j_{N}}^{\dagger}\left(t_{N}\right) \ldots \hat{w}_{j_{1}}^{\dagger}\left(t_{1}\right) \hat{w}_{i_{1}}\left(t_{1}\right) \ldots \hat{w}_{i_{N}}\left(t_{N}\right) \hat{R}(0)\right] .
$$

If the weak entanglement criterion is fulfilled (28) then all of the operators corresponding to the same time which fulfill $i_{k}=j_{k}$ will cancel each other out, so the two functions (30) which are the same for separable evolutions, will always be the same for weakly entangled ones as well.

On the other hand, the set of pairs of functions of type (30) which are the same for separable evolutions is a subset of the set of pairs of such functions which fulfill the commutation criterion (28). Hence, it should be fairly easy to devise a scenario which tests this commutation criterion, e. g. the spin echo $[60,61]$ yields perfect results only if the conditional operators commute. This together with a QEE witness testing the separability criterion (27) would enable to unambiguously distinguish between separable evolutions for which the conditional operators do not commute and strongly entangled evolutions. Yet the ambiguity is still left over in the set of evolutions for which the operators do commute, and distinguishing between such entangling and non-entangling evolutions, although possible on measurements on the environment, is most likely not possible by measurements on the qubit. 


\section{References}

1. Hill, S., Wootters, W.K.: Entanglement of a pair of quantum bits. Phys. Rev. Lett. 78, 5022 (1997). https://doi.org/10.1103/PhysRevLett.78.5022

2. Wootters, W.K.: Entanglement of formation of an arbitrary state of two qubits. Phys. Rev. Lett. 80, 2245 (1998). https://doi.org/10.1103/PhysRevLett.80.2245

3. Yu, T., Eberly, J.H.: Finite-time disentanglement via spontaneous emission. Phys. Rev. Lett. 93, 140404 (2004). https://doi.org/10.1103/PhysRevLett.93.140404

4. Vidal, G., Werner, R.F.: Computable measure of entanglement. Phys. Rev. A 65, 032314 (2002). https:// doi.org/10.1103/PhysRevA.65.032314

5. Lee, J., Kim, M.S., Park, Y.J., Lee, S.: Partial teleportation of entanglement in a noisy environment. J. Modern Optic. 47(12), 2151 (2000). https://doi.org/10.1080/09500340008235138

6. Plenio, M.B.: Logarithmic negativity: a full entanglement monotone that is not convex. Phys. Rev. Lett. 95, 090503 (2005). https://doi.org/10.1103/PhysRevLett.95.090503

7. Eisert, J., Plenio, M.B.: Quantum and classical correlations in quantum brownian motion. Phys. Rev. Lett. 89, 137902 (2002). https://doi.org/10.1103/PhysRevLett.89.137902

8. Hilt, S., Lutz, E.: System-bath entanglement in quantum thermodynamics. Phys. Rev. A 79, 010101 (2009). https://doi.org/10.1103/PhysRevA.79.010101

9. Pernice, A., Strunz, W.T.: Decoherence and the nature of system-environment correlations. Phys. Rev. A 84, 062121 (2011). https://doi.org/10.1103/PhysRevA.84.062121

10. Salamon, T., Roszak, K.: Entanglement generation between a charge qubit and its bosonic environment during pure dephasing: Dependence on the environment size. Phys. Rev. A 96, 032333 (2017). https:// doi.org/10.1103/PhysRevA.96.032333

11. Bonk, F.A., Sarthour, R.S., deAzevedo, E.R., Bulnes, J.D., Mantovani, G.L., Freitas, J.C.C., Bonagamba, T.J., Guimarães, A.P., Oliveira, I.S.: Quantum-state tomography for quadrupole nuclei and its application on a two-qubit system. Phys. Rev. A 69, 042322 (2004). https://doi.org/10.1103/ PhysRevA.69.042322

12. Filipp, S., Maurer, P., Leek, P.J., Baur, M., Bianchetti, R., Fink, J.M., Göppl, M., Steffen, L., Gambetta, J.M., Blais, A., Wallraff, A.: Two-qubit state tomography using a joint dispersive readout. Phys. Rev. Lett. 102, 200402 (2009). https://doi.org/10.1103/PhysRevLett.102.200402

13. Liu, W.T., Zhang, T., Liu, J.Y., Chen, P.X., Yuan, J.M.: Experimental quantum state tomography via compressed sampling. Phys. Rev. Lett. 108, 170403 (2012). https://doi.org/10.1103/PhysRevLett.108. 170403

14. Helm, J., Strunz, W.T.: Quantum decoherence of two qubits. Phys. Rev. A 80, 042108 (2009). https:// doi.org/10.1103/PhysRevA.80.042108

15. Crow, D., Joynt, R.: Classical simulation of quantum dephasing and depolarizing noise. Phys. Rev. A 89, 042123 (2014). https://doi.org/10.1103/PhysRevA.89.042123

16. Viola, L., Lloyd, S.: Dynamical suppression of decoherence in two-state quantum systems. Phys. Rev. A 58, 2733 (1998). https://doi.org/10.1103/PhysRevA.58.2733

17. Viola, L., Knill, E.: Robust dynamical decoupling of quantum systems with bounded controls. Phys. Rev. Lett. 90, 037901 (2003). https://doi.org/10.1103/PhysRevLett.90.037901

18. Calarco, T., Datta, A., Fedichev, P., Pazy, E., Zoller, P.: Spin-based all-optical quantum computation with quantum dots: Understanding and suppressing decoherence. Phys. Rev. A 68, 012310 (2003). https://doi.org/10.1103/PhysRevA.68.012310

19. Economou, S.E., Sham, L.J., Wu, Y., Steel, D.G.: Proposal for optical U(1) rotations of electron spin trapped in a quantum dot. Phys. Rev. B 74, 205415 (2006). https://doi.org/10.1103/PhysRevB.74. 205415

20. Economou, S.E., Reinecke, T.L.: Theory of fast optical spin rotation in a quantum dot based on geometric phases and trapped states. Phys. Rev. Lett. 99, 217401 (2007). https://doi.org/10.1103/ PhysRevLett.99.217401

21. Roszak, K., Filip, R., Novotný, T.: Decoherence control by quantum decoherence itself. Sci. Rep 5, 9796 (2015). https://doi.org/10.1038/srep09796

22. Roszak, K., Marcinowski, L., Machnikowski, P.: Decoherence-enhanced quantum measurement of a quantum-dot spin qubit. Phys. Rev. A 91, 032118 (2015). https://doi.org/10.1103/PhysRevA.91. 032118

23. Mierzejewski, M., Bonča, J., Dajka, J.: Reversal of relaxation due to a dephasing environment. Phys. Rev. A 91, 052112 (2015). https://doi.org/10.1103/PhysRevA.91.052112 
24. Roszak, K., Cywiński, L.: Characterization and measurement of qubit-environment-entanglement generation during pure dephasing. Phys. Rev. A 92, 032310 (2015). https://doi.org/10.1103/PhysRevA. 92.032310

25. Roszak, K.: Criteria for system-environment entanglement generation for systems of any size in puredephasing evolutions. Phys. Rev. A 98, 052344 (2018). https://doi.org/10.1103/PhysRevA.98.052344

26. Roszak, K., Cywiński, L.: Equivalence of qubit-environment entanglement and discord generation via pure dephasing interactions and the resulting consequences. Phys. Rev. A 97, 012306 (2018). https:// doi.org/10.1103/PhysRevA.97.012306

27. Chen, H.B., Gneiting, C., Lo, P.Y., Chen, Y.N., Nori, F.: Simulating open quantum systems with hamiltonian ensembles and the nonclassicality of the dynamics. Phys. Rev. Lett. 120, 030403 (2018). https://doi.org/10.1103/PhysRevLett.120.030403

28. Chen, H.B., Lo, P.Y., Gneiting, C., Bae, J., Chen, Y.N., Nori, F.: Quantifying the nonclassicality of pure dephasing. Nature Comm. 10, 3794 (2019). https://doi.org/10.1038/s41467-019-11502-4

29. Roszak, K.: A measure of qubit environment entanglement for pure dephasing evolutions (2019) arXiv: 1912.07317

30. Zurek, W.H.: Decoherence, einselection, and the quantum origins of the classical. Rev. Mod. Phys. 75, 715 (2003). https://doi.org/10.1103/RevModPhys.75.715

31. Hornberger, K.: Introduction to decoherence theory. Lect. Notes Phys. 768, 221 (2009). https://doi. org/10.1007/978-3-540-88169-8_5

32. Roszak, K., Machnikowski, P.: Complete disentanglement by partial pure dephasing. Phys. Rev. A 73, 022313 (2006). https://doi.org/10.1103/PhysRevA.73.022313

33. Biercuk, M.J., Uys, H., VanDevender, A.P., Shiga, N., Itano, W.M., Bollinger, J.J.: Optimized dynamical decoupling in a model quantum memory. Nature 458, 996 (2009). https://doi.org/10.1038/nature07951

34. Bylander, J., Gustavsson, S., Yan, F., Yoshihara, F., Harrabi, K., Fitch, G., Cory, D.G., Nakamura, Y., Tsai, J.S., Oliver, W.D.: Dynamical decoupling and noise spectroscopy with a superconducting flux qubit. Nat. Phys. 7, 565 (2011). https://doi.org/10.1038/nphys1994

35. Medford, J., Cywiński, Ł., Barthel, C., Marcus, C.M., Hanson, M.P., Gossard, A.C.: Scaling of dynamical decoupling for spin qubits. Phys. Rev. Lett. 108, 086802 (2012). https://doi.org/10.1103/ PhysRevLett.108.086802

36. Staudacher, T., Shi, F., Pezzagna, S., Meijer, J., Du, J., Meriles, C.A., Reinhard, F., Wrachtrup, J.: Nuclear magnetic resonance spectroscopy on a (5-nanometer) ${ }^{3}$ sample volume. Science 339, 561 (2013). https://doi.org/10.1126/science.1231675

37. Roszak, K., Mazurek, P., Horodecki, P.: Anomalous decay of quantum correlations of quantum dot qubits. Phys. Rev. A 87, 062308 (2013). https://doi.org/10.1103/PhysRevA.87.062308

38. Muhonen, J.T., Dehollain, J.P., Laucht, A., Hudson, F.E., Kalra, R., Sekiguchi, T., Itoh, K.M., Jamieson, D.N., McCallum, J.C., Dzurak, A.S., Morello, A.: Storing quantum information for 30 seconds in a nanoelectronic device. Nat. Nanotechnol. 9, 986 (2014). https://doi.org/10.1038/nnano.2014.211

39. Malinowski, F.K., Martins, F., Cywiński, Ł., Rudner, M.S., Nissen, P.D., Fallahi, S., Gardner, G.C., Manfra, M.J., Marcus, C.M., Kuemmeth, F.: Spectrum of the nuclear environment for GaAs spin qubits. Phys. Rev. Lett. 118, 177702 (2017). https://doi.org/10.1103/PhysRevLett.118.177702

40. Szańkowski, P., Ramon, G., Krzywda, J., Kwiatkowski, D., Cywiński, L.: J. Environmental noise spectroscopy with qubits subjected to dynamical decoupling. Phys.: Condens. Matter 29(33), 333001 (2017). https://doi.org/10.1088/1361-648X/aa7648

41. Roszak, K., Kwiatkowski, D., Cywiński, L.: How to detect qubit-environment entanglement generated during qubit dephasing. Phys. Rev. A 100, 022318 (2019). https://doi.org/10.1103/PhysRevA.100. 022318

42. Kwiatkowski, D., Cywiński, L.: Decoherence of two entangled spin qubits coupled to an interacting sparse nuclear spin bath: Application to nitrogen vacancy centers. Phys. Rev. B 98, 155202 (2018). https://doi.org/10.1103/PhysRevB.98.155202

43. Wrachtrup, J., Finkler, A.: Single spin magnetic resonance. J. Magn. Res. 369, 225 (2016). https://doi. org/10.1016/j.jmr.2016.06.017

44. Degen, C.L., Reinhard, F., Cappellaro, P.: Quantum sensing. Rev. Mod. Phys. 89, 035002 (2017). https://doi.org/10.1103/RevModPhys.89.035002

45. Borri, P., Langbein, W., Schneider, S., Woggon, U., Sellin, R.L., Ouyang, D., Bimberg, D.: Ultralong dephasing time in InGaAs quantum dots. Phys. Rev. Lett. 87, 157401 (2001). https://doi.org/10.1103/ PhysRevLett.87.157401 
46. Vagov, A., Axt, V.M., Kuhn, T.: Impact of pure dephasing on the nonlinear optical response of single quantum dots and dot ensembles. Phys. Rev. B 67, 115338 (2003). https://doi.org/10.1103/PhysRevB. 67.115338

47. Vagov, A., Axt, V.M., Kuhn, T., Langbein, W., Borri, P., Woggon, U.: Nonmonotonous temperature dependence of the initial decoherence in quantum dots. Phys. Rev. B 70, 201305 (2004). https://doi. org/10.1103/PhysRevB.70.201305

48. Roszak, K., Machnikowski, P.: "Which path" decoherence in quantum dot experiments. Phys. Lett. A 351(4-5), 251 (2006). https://doi.org/10.1016/j.physleta.2005.11.012

49. London, P., Scheuer, J., Cai, J.M., Schwarz, I., Retzker, A., Plenio, M.B., Katagiri, M., Teraji, T., Koizumi, S., Isoya, J., Fischer, R., McGuinness, L.P., Naydenov, B., Jelezko, F.: Detecting and polarizing nuclear spins with double resonance on a single electron spin. Phys. Rev. Lett. 111, 067601 (2013). https://doi.org/10.1103/PhysRevLett.111.067601

50. Fischer, R., Bretschneider, C.O., London, P., Budker, D., Gershoni, D., Frydman, L.: Bulk nuclear polarization enhanced at room temperature by optical pumping. Phys. Rev. Lett. 111, 057601 (2013). https://doi.org/10.1103/PhysRevLett.111.057601

51. Wunderlich, R., Kohlrautz, J., Abel, B., Haase, J., Meijer, J.: Optically induced cross relaxation via nitrogen-related defects for bulk diamond ${ }^{13} \mathrm{C}$ hyperpolarization. Phys. Rev. B 96, 220407 (2017). https://doi.org/10.1103/PhysRevB.96.220407

52. Scheuer, J., Schwartz, I., Müller, S., Chen, Q., Dhand, I., Plenio, M.B., Naydenov, B., Jelezko, F.: Robust techniques for polarization and detection of nuclear spin ensembles. Phys. Rev. B 96, 174436 (2017). https://doi.org/10.1103/PhysRevB.96.174436

53. Poggiali, F., Cappellaro, P., Fabbri, N.: Measurement of the excited-state transverse hyperfine coupling in NV centers via dynamic nuclear polarization. Phys. Rev. B 95, 195308 (2017). https://doi.org/10. 1103/PhysRevB.95.195308

54. Pagliero, D., Rao, K.R.K., Zangara, P.R., Dhomkar, S., Wong, H.H., Abril, A., Aslam, N., Parker, A., King, J., Avalos, C.E., Ajoy, A., Wrachtrup, J., Pines, A., Meriles, C.A.: Multispin-assisted optical pumping of bulk ${ }^{13} \mathrm{C}$ nuclear spin polarization in diamond. Phys. Rev. B 97, 024422 (2018). https:// doi.org/10.1103/PhysRevB.97.024422

55. Hovav, Y., Naydenov, B., Jelezko, F., Bar-Gill, N.: Low-field nuclear polarization using nitrogen vacancy centers in diamonds. Phys. Rev. Lett. 120, 060405 (2018). https://doi.org/10.1103/ PhysRevLett.120.060405

56. Mahan, G.D.: Many-Particle Physics. Kluwer, New York (2000). https://doi.org/10.1007/978-1-47575714-9

57. Grodecka, A., Jacak, L., Machnikowski, P., Roszak, K.: Phonon impact on the coherent control of quantum states in semiconductor quantum dots. in Quantum Dots: Research Developments (Nova Science, NY, 2005), p. 47. arXiv:cond-mat/0404364

58. Andriambololona, R.: A diagonalization method of a hamiltonian in second-quantization. I. Prog. Theor. Phys. 41(4), 1064 (1969). https://doi.org/10.1143/PTP.41.1064

59. Kwiatkowski, D., Szańkowski, P., Cywiński, L.: Influence of nuclear spin polarization on the spin-echo signal of an NV-center qubit. Phys. Rev. B 101, 155412 (2020). https://doi.org/10.1103/PhysRevB. 101.155412

60. Herzog, B., Hahn, E.L.: Transient nuclear induction and double nuclear resonance in solids. Phys. Rev. 103, 148 (1956). https://doi.org/10.1103/PhysRev.103.148

61. Gordon, J.P., Bowers, K.D.: Microwave spin echoes from donor electrons in silicon. Phys. Rev. Lett. 1, 368 (1958). https://doi.org/10.1103/PhysRevLett.1.368

Publisher's Note Springer Nature remains neutral with regard to jurisdictional claims in published maps and institutional affiliations. 